(2) It is necessary to take into account the fact that there are probably temperature fluctuations in the photospheres of other stars as well as the Sun. Such fluctuations may play a major role in the interpretation of Dr Wright's results on the apparent correlation between turbulent velocity and excitation potential. Imagine, for example, a situation in which a group of lines are strong in a 'cold' region and weak, or absent, in a 'hot' region. The effect on the total equivalent width is roughly similar to that produced by scattered light in the spectrograph. All values of the equivalent width are reduced by a constant multiplying factor and the result is an apparent decrease in turbulent velocity. But the half-widths are not changed and hence the turbulent velocity derived from the curve of growth is less than that from the profile. Also, of course, the modification of the curve of growth will be different for lines of different ionization and excitation potential.

Dr Greenstein:

I believe that a more sophisticated view of the velocity field in the giant star's reversing layer is needed. It is known that there is about 2 or $3 \mathrm{~km}$. $/ \mathrm{sec}$. differential velocity between excited and resonance lines in ordinary red giants. In supergiants, line-profiles change, and even become double. The effectiveness of a velocity gradient in elevating a curve of growth is like that of turbulence.

I would suggest the usefulness of a two-stream model, before consideration of whether we should adopt fully developed aerodynamical turbulence.

I would also like to know whether the macro- or micro-turbulence velocity is the relevant one in computation of the effective support of the reversing layer by turbulence.

Dr Minnaert, in reply to Dr Greenstein's last question:

There is no doubt that micro-turbulence produces turbulent expansion. I would not dare to say definitively that macro-turbulence plays the same role. In a private conversation Dr McCrea, who suggested this effect many years ago, expressed the opinion that both should be treated in the same way.

(Note added after the meeting.) An argument favouring that last-meritioned opinion is that micro- and macro-turbulence differ only when we compare the size of their elements to the thickness of the transparent atmospheric layers; but that is a question of pure optics, and it is clear that it has nothing to do with the turbulent expansion, which is a purely mechanical effect.

Dr Unsöld, in reply to Dr Greenstein:

(I) The largest turbulence elements should have diameters of the order of the atmosphere's scale height. Therefore we should expect macro-turbulence in addition to microturbulence, if the optical depth corresponding to one scale height $H$ is $\kappa H>I$. Calculations made by K. H. Böhm (Physik der Sternatmosphären, p. 462) indicate that for normal atmospheres of any temperature and surface gravitation $\kappa H$ is, by chance, practically always of the order of unity. Macro-turbulence should therefore play a major role only in atmospheres, e.g., made up of huge prominences, etc.

(2) In general, quite good accuracy can be obtained using the 'schematic' curve of growth in connexion with the theory of weight functions for calculating the effective number of atoms (or optical depth) for weak absorption. Aiming at higher accuracy, one must in many cases also take into account the variation of turbulent velocities with height, the inhomogeneity of the atmosphere, and other effects which complicate the subject enormously. It does not help to increase the accuracy only in one direction!

\title{
3. INTERPRETATION OF THE MOTIONS IN STELLAR ATMOSPHERES
}

\section{By L. Brermann, Max Planck Institut für Physik, Göttingen}

In a theoretical interpretation of the motions observed in stellar atmospheres, which are often characterized as turbulent, it seems fitting to start by stating more precisely the essential physical features of turbulence.

For this it is convenient to regard the velocity field decomposed into its Fourier 
components, separately for each cartesian component of the velocity, with the wave number $\left(\mathrm{cm}^{-1}\right)$ as argument. In this description one may say that energy, put into the system at small wave numbers, is transmitted to successively larger wave numbers by the action of the non-linear terms of the equations of hydrodynamics, to be finally dissipated into heat at some range of large wave numbers( $x$. The input of energy may be effected by a dynamic instability of the velocity field, or else by unequal heating causing thermal instability -in this case one may speak of convection, in contrast to dynamical turbulence-but for the wave numbers in the intermediate range this is of no great consequence. This description applies strictly only to an incompressible fluid, but it still is a reasonable approximation in the case of subsonic velocities. In the range of sonic and supersonic velocities, the formation of shock waves and fronts, in which most of the dissipation takes place, modifies this picture somewhat; but it is probably mainly a question of terminology whether (or not) one uses the term turbulence also for this case ( 2 ).

Under experimental conditions the regions of space, where the energy is put into the system, often coincide with those in which the energy is dissipated. But in discussing the motions in stellar atmospheres it seems essential to distinguish between the former regions, in which energy is deviated from the radiative flow of energy, and the latter ones, in which this energy is ultimately again dissipated into heat, and to consider in addition the possibility that there are regions of space through which the energy is possibly only transmitted by travelling waves, without much loss by dissipation into heat.

In the case of the Sun and similar stars the following tentative and very schematic picture may perhaps serve as a background to our discussion:

Underneath the visible layers of the photosphere we have the hydrogen convection zone, about which I am going to say more in a minute. It is characterized by thermal instability, the heat flux maintains the actual temperature gradient at a level above the so-called adiabatic gradient, thereby causing a stationary turbulent state of motion with a turbulent velocity of $\approx I-2 \mathrm{~km}$. $/ \mathrm{sec}$. near the surface. The largest part of the energy thus put into the system is dissipated also in this zone. But a certain, probably small, fraction of it escapes dissipation by giving rise to travelling waves, a part of which travels from the unstable layers of the hydrogen convection zone into the outer atmosphere (3).

Above the photosphere and below the upper chromosphere there is probably an intermediate region, the upper boundary of which cannot yet be indicated more precisely, which is probably pervaded by these travelling waves - a kind of acoustic noise-without much loss of energy. If this is so, the term turbulence, according to what has been said before, would not apply to the state of motion of these layers. The distinction seems to be a relevant one, since it is connected with an essential point of the theory of these layers (cf. the subsequent remarks concerning the formation of spicules).

The relative amplitude of the travelling waves in question must increase outwards since the density decreases outwards very strongly. At that layer, in which the velocity amplitude $\Delta v$ becomes comparable with the velocity of sound $v_{s}$, shock waves must arise, and from thereon the dissipation must become effective. This region, which might again be called turbulent from the point of view we have taken above, is tentatively identified with the medium and the upper chromosphere; however, a certain part of the remaining energy, according to the theoretical pictures under discussion since I947, escapes into the corona and maintains its energy balance, both with regard to its radiative and its corpuscular emissions which seem quite comparable in amount.

We discuss now more in detail the properties of the hydrogen convection zone (4), the instability of which is caused by the effect of the increase (in the inward direction) of the opacity, and in those layers, in which the degree of ionization of the hydrogen is appreciably different both from 0 and from $I$, also by the larger value of the specific heat. When this zone was first discussed by Unsöld 25 years ago, the contribution of the turbulence to the transport of heat and its influence on the distribution of the density was thought negligible, in accordance with the estimates of the photospheric pressure current at 
that time $\left(\approx\right.$ roo dyn. $\left./ \mathrm{cm}^{2}{ }^{2}\right)$. Around $x 938$ it was found, however, that the pressure in the Sun's photosphere is very much larger, and an application of the theory of convective transfer of heat indicated then that its contribution to the transport of energy is not at all small and probably causes the convection zone to have a depth very much in excess of that resulting from the first picture, being perhaps of the order of a tenth of the solar radius, with a temperature of some million degrees at the inner boundary.

The characteristic lengths relevant to the discussion of motions in stellar atmospheres are therefore at least two: first the depth of the zone itself, second the scale height $R T / \mu g$ ( $R$ gas constant, $T$ temperature, $\mu$ molecular weight, $g$ gravitational acceleration) near its outer boundary, which is of the order of Ioo km. on the Sun for the subphotospheric level with $T \approx 6000-8000^{\circ}$. The value of the turbulent velocity $v$ may be found by combining the equation of heat transfer by turbulence or convection, which may be written (with $c_{p}$ specific heat per gram at constant pressure, $p_{g}$ gas pressure, $\Delta T$ mean temperature difference between an element of turbulence and its surrounding)

$$
H_{k} \approx\left(\frac{c_{p} \mu}{R}\right) \times p_{g} \times v \times \frac{\Delta T}{T}\left[\frac{\mathrm{erg}}{\mathrm{cm} .^{2} / \mathrm{sec} .}\right],
$$

with the equation expressing the balance of energy

$$
v^{2} \approx g l \frac{\Delta T}{T}
$$

(with possibly a correction factor of $\approx \frac{1}{2}$ on the right-hand side, $l$ being the so-called mixing length, an analogon to the mean free path of kinetic theory introduced by L. Prandtl).

In the case of the Sun and similar stars, the resulting velocity (found by elimination of $\Delta T / T)$ turns out to be small in any case compared with the velocity of sound. Under these conditions the scale height, or a small multiple of it, should be regarded as a reasonable estimate for the mixing length $l$ and, at the same time, for the diameter of the elements; the corresponding velocity is found to be between $\mathrm{I}$ and $2 \mathrm{~km}$. $/ \mathrm{sec}$. The most detailed recent work on the solar hydrogen convection zone is that due to Mrs Böhm-Vitense (4).

When these values are compared with the observations of solar granulation as reviewed by Dr de Jager, the velocity seems to be in accord with the theoretical expectation, whereas the dimensions of the granules are usually found to be of the order of 700 or rooo $\mathrm{km}$., that is, several times larger than the theory leads us to expect. This might be an indication that the theory is still too crude in its present form; alternatively, the explanation may well be that the observed size actually reflects only the influence of the limitations imposed by the observing conditions rather than the true size of the granules themselves.

Owing to the influence of the gravitational field, the turbulence cannot be isotropic in the scale $l$; only the sub-turbulence connected with larger wave numbers should be isotropic to a higher degree.

The case of the Sun shows also the importance of another feature of the physical processes going on in the hydrogen convection zone, that is, the creation of acoustic noise, which escapes into the outer layers $(2,3)$. If the velocity field in the hydrogen convection zone is decomposed into its curl-free and its divergence-free parts, it is only the former which causes the travelling waves; in addition, in a gravitational field there is a certain critical frequency with the property that only frequencies above the critical one can belong to travelling waves (the subcritical ones lead to other types of wave motion). The energy flux connected with this noise is given by the usual expression $\rho(\Delta v)^{2} \times v_{s}(\rho$ mass density, $\Delta v$ velocity amplitude connected with the noise) as long as $\Delta v / v_{s}$ is smaller than unity. If it becomes of order unity, we have to a first approximation simply $\rho\left(v_{s}\right)^{3}$. Simultaneously, owing to the formation of shock waves, the dissipation of this acoustic energy must become appreciable, and probably conditions are such that, 
in spite of the decrease in the outward direction of the acoustic energy flux, the temperature maintained by the dissipation of acoustic energy increases outwards.

The presence of magnetic fields gives rise to the appearance of a new class of phenomena, those of hydromagnetics, two features of which are: a new characteristic velocity $v_{A}$, discovered by Alfvén(6); and an anisotropy of the velocity of propagation of waves, when $v_{A}$ is comparable to or larger than $v_{s}$. Outside spots, this happens in the upper chromosphere (and the corona) of the Sun.

The formation of spicules in this region has probably to be understood as a consequence of the presence of photospheric magnetic fields. Schwarzschild and Schlüter have started an investigation of this hydromagnetic phenomenon, the preliminary results of which seem promising. But little can be said at present, I am afraid, with respect to the more detailed features described by Dr de Jager.

The amount of energy going into noise should be referred to the energy dissipated by turbulence per gram of matter and per second, which itself is given by the expression $v^{3} / l$. Recent work of Lighthill, of Proudman and of others $(5,2)$ indicates that the fraction of energy which escapes dissipation by becoming mechanical energy of sound, depends itself in a very sensitive way on the ratio of the turbulent velocity $v$ to the velocity of sound, that is to say, on the Mach number $M$ connected with $v$. In an ideal case studied by Lighthill and Proudman, which is characterized by the neglect of the influence of the finite value of the scale height, the effectivity of the noise production is of the order of the $5^{\text {th }}$ power of $M$ with a numerical coefficient of the order IO $^{1}$. Now closer inspection reveals that the exponent is certainly on the high side and that a value, say between 3 and 4 , is more likely to be true; also magnetic fields, according to recent work of Kulsrud, influence the amount of energy going into noise considerably.

Combining the expression for the dissipation of energy with the effectivity coefficient, it is seen that in any case the energy going into noise depends on a very high power of the turbulent velocity, somewhere between 6 and 8 . If the chromosphere and the solar corona derive their energy from this noise, the chromospheric and coronal activity should depend in the same way on the turbulent velocity. It is therefore not surprising that one observes considerable variations in the extent of these layers on the Sun, whereas so far the connected variations in the turbulent velocity have escaped observation. But certainly this connexion is one to which one should, if possible, pay some attention.

In the study of stellar atmospheres one distinction which seems often of importance is that between micro-turbulence and macro-turbulence. The former reveals itself from the curve of growth, whereas the latter may be obtained from the line-profiles; the dividing scale of length is obviously the mean free path of light in the atmosphere (or a small multiple of it). This distinction is relevant especially in the case of giant and supergiant stars, where the equations stated before lead to velocities to the order of the velocity of sound. Since their combination yields

$$
g l H_{k}=\left(\frac{c_{p} \mu}{R}\right) p_{g} v^{3}
$$

where $g l$ on the left-hand side, as mentioned before, may be regarded as fixed by the temperature near the outer boundary of the hydrogen convection zone, the increase of the velocity with decreasing surface pressure is comparatively slow, but since $p_{g}$ is roughly $\sim \sqrt{ } g$, turbulent velocities of the order of the velocity of sound must appear and are actually observed on giants and supergiants as we have seen from Dr Wright's report.

However, if the equations as given so far lead to a value $v$ exceeding the velocity of sound $v_{s}$, the assumptions on which these equations are based(4) are no longer well justified. In this case especially, the second relation would seem to justify only the conclusion that $v$ and $v_{s}$ are of the same order of magnitude (say equal, disregarding a factor of $\mathrm{I}$ or $\pi$ ), $v_{s}$ thus being a kind of effective upper limit to $v$, while $\Delta T / T$ should then also be of order unity. The equation of convective transfer, applied to a typical giant, would then lead to the conclusion that, since only at some distance from the outer boundary of the order of some scale heights $H_{k}$ would become comparable with the 
actual heat flux, $v$ would first increase inwards with $v_{s}$, to decrease again only after $c_{p} \rho$ (density) has become sufficiently large to allow $v$ to drop below $v_{s}$.

Under these circumstances no clear distinction can be made between the convective transfer of heat and the acoustic propagation of energy, and the whole range of the values of $v$ may be expected to manifest itself on the surface, that is to say, differences between macro- and micro-turbulence seem plausible, as they are actually found on some stars of this class.

The observations as discussed by Dr Wright seem to indicate much less uniformity in a given region of the Hertzsprung-Russell diagram than would be expected from what has been said so far. It should be added therefore that the characteristics of the hydrogen convection zone should, from a theoretical point of view, depend also on several other factors, in particular the chemical composition and the state of rotation. Theory shows that at least for stars of solar type the depth of the convective zone may be sensitive to the conditions just mentioned. In addition the magnetic fields, which are probably connected with the turbulence, and also larger-scale fields, have to be thought of ; but little can be said so far about what their influence is likely to be.

Reviewing finally the main sequence stars of early type, there is a two-fold difference against the case of the Sun: first, the photospheric pressure is smaller by about 3 powers of ro; secondly, the heat flux flowing through the atmosphere is much larger. The effect of both is to make the contribution of the turbulence to the energy transport small compared with the radiative mechanism of transport over a large range of the pressure. Therefore the convective zone of these stars should be only shallow, and we should expect turbulent velocities of the order of the velocity of sound, but no difference between microand macro-turbulence. This expectation is, apparently, at least not contradicted by the observations according to Dr Wright's discussion.

The production of acoustic noise is probably the most effective mechanism leading ultimately to the output of non-thermal energy. The various non-thermal forms in which energy is emitted by stars have recently received considerable atiention ( 7$)$; the most important ones are electro-magnetic radiation in the radio frequency range and in the U.v. and Röntgen range, and corpuscular emissions with a velocity of the order of the escape velocity from the surface (around rooo $\mathrm{km}$. $/ \mathrm{sec}$. for the Sun), and last the acceleration of charged particles to cosmic ray energies. The considerations put forward in the foregoing would then seem to lead to the expectation that stars with high velocities of turbulence at the surface should emit with a much higher efficiency the non-thermal radiations and emissions just mentioned, in contrast to the Sun, on which the fraction of energy emitted non-thermally is probably quite small (around $\mathrm{ro}^{-6}$, taking an overall average).

The theoretical picture we have arrived at is simple therefore in so far the hydrogen convection zone appears to be the dominating cause of all types of motions in stellar atmospheres. On the other hand, the theoretical interpretation of its detailed features is certainly still in its beginnings, and much work both with regard to the observations and the theory will be needed for a fuller understanding.

\section{REFERENCES}

(1) Kolmogoroff, C.R. Acad. Sci. U.S.S.R. 30, 30I; 32, I6, 194I.

Prandtl u. Wieghardt, Nachr. d. Akad. d. Wiss. Göttingen, Math-Phys. Klasse, S. 6, I945.

Onsager, Phys. Rev. Dez. I945.

v. Weizsäcker, $Z$. f. Physik, 124, 614, I948. and especially

Heisenberg, Z. f. Physik, 124, 628, r948.

(2) Cf. Lighthill, in: Gas Dynamics in Interstellar Clouds (I.A.U. Symposium, Series no. 2, r955, ed. by H. C. v. d. Hulst and J. M. Burgers), p. I21. 
(3) Schwarzschild, Ap. J. 107, I, 1948.

Biermann, Naturwiss, 33, I 8 , 1946.

Biermann, $Z . A p .25,16 \mathrm{I}, 1948$.

Schatzman, $A n n . A p$. 12, 203, 1949.

(4) Unsöld, $Z$. Ap. 1, 138; 2, 209, I931.

Prandtl, Beitr. z. Phys. d. fr. Atmosph. 19, I88, 9932.

Biermann, $Z$. $A p .5$, I I $7,1932$.

Biermann, $Z$. $A p .21,320 ; 22$,244, 1942.

Böhm-Vitense, $Z$. Ap. 32, I35, 1953.

Biermann, Naturwiss. 21, 2, 1945.

Unsöld, Physik der Sternatmosphären, 2. Auflage, 1955, VIII. Kap.

(5) Lighthill, Proc. Roy. Soc. A. 211, 564, r952.

Proudman, Proc. Roy. Soc. A. 214, I I9, 1952.

Kulsrud, $A p . J$. 121, 46I, 1955.

(6) Alfvén, Ark. mat. fys. 29 B, no. 2, 1942.

Alfvén, Cosmical Electrodynamics, Oxford, 1950.

Lundquist, Ark. Fys. 5, no, 15, 1952.

(7) Cf. e.g.

Biermann, in: Gas Dynamics in Interstellar Clouds (I.A.U. Symposium, Series no. 2, 1955, ed. by H. C. v. d. Hulst and J. M. Burgers), p. 2 I 2.

Dr Thomas:

\section{Discussion}

It seems to me that in any attempt to give a physical interpretation of the rather heterogeneous collection of astrophysical observations underlying what we call turbulence in stellar atmospheres, three aspects should be stressed.

First, we should be very careful to distinguish between a discussion of those velocity fields which we predict, and an interpretation of those velocity fields which we observe. It would be encouraging, indeed, from the standpoint of our physical understanding of stellar atmospheres if these two categories were identical. Unfortunately such identity is not obvious at all. It seems to me that Dr Biermann has presented a discussion from the standpoint of the first category; an excellent summary of current thinking and work on the velocity field arising from the hydrogen convection zone, and its particular application to the velocities associated with the solar granulation. At such a symposium as this, particularly with the title of this third paper, one should, however, note that a large number of the observed motions in stellar atmospheres do not seem explained by this simple recourse to convective motion and its later amplification by the atmospheric density gradient. Specifically, from Dr Biermann's remarks, it follows that by the mechanism discussed one can hardly expect to generate material velocities much in excess of the thermal velocity. Yet one of the most interesting features of 'turbulence' in stellar atmospheres has been the observations of velocities apparently exceeding the local thermal velocity by factors of 2, 3 and higher. We can hardly ignore these velocity fields.

Secondly, we have the problem which must be settled before we can progress far in the actual physical interpretation of motions in stellar atmospheres-that of simply describing, in a self-consistent aerodynamical way, the observed motions. We have heard today both expressions of dissatisfaction at the use of the term 'turbulence' for the categoric description of motions in stellar atmospheres, and a plea that we engage not in a semantic discussion but stick to physics. My own feeling has always been that the semantic difficulty is a very real one indeed; for, in spite of all protestations that astronomical turbulence is not to be confused with the phenomena of laboratory aerodynamic turbulence, practically all the astronomical interpretive literature on the subject since the war has consisted of a preoccupation with, or an application of, the results of modern developments in laboratory turbulence. It seems to me that the most fruitful subject for investigation in this regard is the extent to which we must treat all motions in stellar 
atmospheres only in terms of a Fourier analysis of the velocity field, and the extent to which we can describe at least some of these motions in terms of a gas stream with a local systematic velocity in addition to a random component. I would like to concur very heartily in Dr Greenstein's remark, after Dr Wright's presentation, that the terms 'macro-turbulence' and 'micro-turbulence' were perhaps unfortunate and should be replaced by a discussion of 'streaming motions'. I venture to predict that the aerodynamic methodology adopted for the approach to the solution of these problems will not be invariant under the semantic-conceptual change. At the same time I am sure that such a result would not prove anything more profound than that we have little understanding of these observed motions in stellar atmospheres and are simply trying to proceed by analogy with what we know of gas dynamics from other sources. And the unfortunate situation is that we have at present too few analytical solutions of gas-flow problems under the thermodynamic conditions holding in stellar atmospheres to be of much guide to our physical intuition.

Thirdly, we have the problem of the coupling between the translational and internal degrees of freedom of the gas. It is chiefly in this situation that the gas-dynamical flow patterns in the astronomical environment distinguishes itself from that in the usual laboratory case. Only small velocity fields are required to change appreciably the state of excitation and ionization of the gas, because the energy level of the gas is already so high. The converse holds in the laboratory. It seems to me that the primary problem associated with the rather high-speed motions observed in stellar atmospheres is just this effect on the internal degrees of freedom; what is it, and why do the results not stand out more than they apparently do? A negligible effect on the continuous spectrum may possibly be understood in terms of the atmospheric level at which the velocities occur. There should, however, be some excitation effect in the line-spectrum, and the interpretation of its magnitude represents a primary problem.

Dr Biermann, in reply to Dr Thomas:

On the whole, I have not the feeling that there is too much disagreement between Dr Thomas and me. With regard to his point one, it is indeed my impression that the major factors which determine the observed features are those mentioned (part of them only too briefly) in my discussion; but I may, of course, be wrong in this, and I certainly agree that those gaps between theoretical expectation and observation, to which $\mathrm{Dr}$ Thomas drew attention, deserve closer study. As to the second point, I would only like to say that it gives additional emphasis to the necessity of future efforts to arrive at a more and more deductive type of theory of turbulence. The third problem seems to be largely that of the interaction of the complex radiation field and the atmospheric matter, but there is little I have to add at this moment.

Dr Schatzman:

(I) The flux of mechanical energy depends on the extension of the hydrogen convective zone. We may expect the mechanical flux larger, when the extension of the hydrogen convective zone is greater. In this connexion we can refer to the remark of Hoyle and Schwarzschild on the existence of turbulent velocities of the order of sound velocities in the atmospheres of red supergiants.

(2) There comes the question of the upper limit of the convective zone. I do not see how the 'turbulent pressure' comes into the expression of the adiabatic compressibility which has to be compared with the radiative compressibility for defining the upper limit of the convective zone. Therefore, I think that, for supergiant stars, the convective region has no real outer boundary, and becomes a complicated region with jets of matter and large hydrodynamical motions.

(3) This leads me to the point so much stressed by Prof. Minnaert. If we have large gaseous masses moving at random with velocities of 20 to $30 \mathrm{~km}$. $/ \mathrm{sec}$, , such masses colliding together produce shock fronts with temperatures as high as $200,000^{\circ}$. Very important spectroscopic effects should result which do not show up. There is a strong contradiction between the observations of the spectra and the predicted phenomena in highly supersonic turbulence. 
Naturally, a phenomenological theory of the transfer of compression waves can be developed, with an equation looking like:

$$
\frac{d\left(\rho v^{2} V_{s}\right)}{d h}=-\frac{\rho v^{3}}{V_{s} \theta}
$$

where $\theta$ is a constant having the dimensions of a time. Such a theory leads to a limit value of the material velocity which may be highly supersonic, but has not much meaning, on account of the above-mentioned contradictions.

(4) It is quite important to measure precisely the total amount of energy radiated away by the corona and the chromosphere. If it is $10^{-3} L$, then, in the photosphere, one has to have a mechanical flux $\rho \bar{v}^{2} V_{s}$ with material velocities $\sqrt{ } \bar{v}^{2} \sim \mathrm{I} \mathrm{km}$. $/ \mathrm{sec}$.; but if it is $10^{-5} L, \sqrt{ } \bar{v}^{2}$ is only $0 \cdot \mathrm{Im}$. $/ \mathrm{sec}$. It would therefore stress the importance of such a quantity for the theory of the solar granulation.

Dr Minnaert:

I should like to illustrate Dr Biermann's lecture by the following schematic drawing

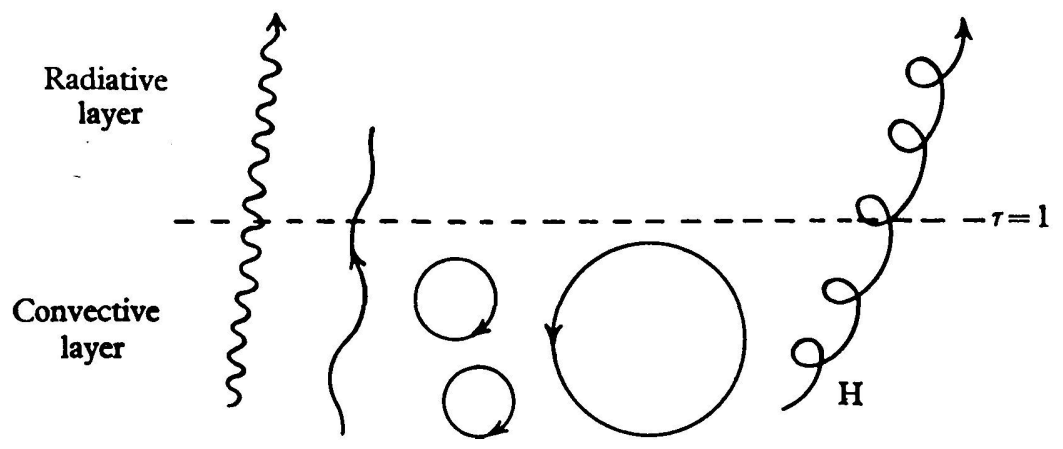

The layer $\tau=\mathrm{I}$ separates the radiative layer from the deeper convective layer. Progressive waves of small wave-length are transmitted through the radiative layer; progressive waves of longer wave-length and rotational motions are not transmitted or quickly damped. Where there are magnetic fields, waves may travel into the radiative region.

Dr Biermann explained what the granules are not. But how must we understand what they are?

Dr Biermann, in reply to Dr Minnaert:

According to the picture which has been developed in my address as the more probable one, the granulae should be identified with the largest elements of turbulence near the upper boundary of the hydrogen convection zone. Whether or not this identification is correct, seems essentially to depend on whether the acoustic energy, escaping dissipation in the hydrogen convection zone itself, is small compared with the total dissipated energy or of comparable amount. If the first alternative represents the actual conditions on the Sun, that is to say, if the $\Delta v$ in the expression of the flux of acoustic energy is really small compared with the velocity $v$ of the largest elements of turbulence, then indeed the granulae are essentially elements of turbulence, which have penetrated into regions of optical depth smaller than but of the order unity, and their quality of being at the same time source of mechanical energy for the chromosphere and the corona may for this purpose be disregarded. If, on the other hand, the picture mentioned several times in this discussion is correct, according to which the stream of acoustic energy is very much larger and $\Delta v$ of the order of $v$ or of the velocity of sound itself already in the photosphere, then the physical situation is much more complex.

Dr Schwarzschild:

(I) For the case of subsonic turbulent velocities, as in the photosphere of the Sun, Dr Biermann has discussed the equations for the flux and for the average velocities. The most uncertain factor in these equations is the free path-length $L$. The value of $L$ 
sensitively affects the depths of the convection zone and this has a noticeable influence on the internal structure of the star. Preliminary calculations for the interior of the Sun suggest that the proper value of $L$ may lie in the neighbourhood of three scale heights.

(2) For the case of supersonic velocities of large elements in supergiants, valuable data might be gained if high-dispersion spectra of one or two bright supergiants could be taken sufficiently often and over a long enough time interval so that measurements of the random time variations of the line-profiles could give statistically significant information regarding the average number of elements covering the stellar surface.

Dr Greenstein:

I wish to remark that the high macroscopic turbulent velocity in supergiants is not apparently accompanied by any large increase in emission-line coronal or chromospheric activity: the dependence of $\mathrm{Ca}$ II emission-line intensity and width on luminosity is definite, but $\mathrm{H} \alpha$ is seldom seen in emission. The width of Ca II self-reversal also increases with luminosity, according to $\mathrm{O}$. C. Wilson. But it is improbable that the $20 \mathrm{~km}$. $/ \mathrm{sec}$. motions shown by absorption-line profiles degenerate into a true kinetic or electron temperature. Only in the W Virginis stars are there strong emission lines, connected with collision of moving layers. 